\title{
Pénurie de médecins: les caisses freinent la médecine de famille
}

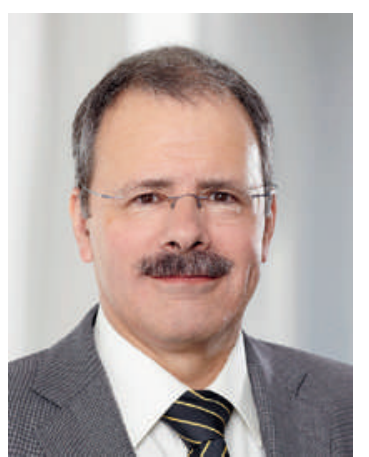

Au printemps prochain, le Parlement devrait se prononcer sur l'initiative parlementaire du conseiller national Olivier Feller consacrée à la «non-discrimination des médecins spécialistes en médecine interne générale titulaires d'un deuxième titre de spécialiste». La FMH soutient clairement cette initiative qui vise à modifier la loi sur l'assurancemaladie afin d'interdire l'exclusion injustifiée des porteurs de deux titres.

Ce problème est lié à l'attitude de certains assureurs qui refusent d'intégrer les spécialistes en médecine interne générale ayant obtenu un deuxième titre de spécialiste (par ex. en allergologie, immunologie, rhumatologie ou endocrinologie) dans leurs listes de médecins de premier recours, au motif qu'ils coûteraient plus cher que leurs collègues qui sont uniquement spécialistes en médecine interne générale lorsqu'ils sont consultés en tant que généralistes. Or aucun assureur n'est en mesure de prouver cette hypothèse.

\section{L'exclusion par les caisses des spécialistes en médecine interne générale porteurs d'un deuxième titre de spécialiste est contraire au principe de \\ l'égalité de traitement.}

Concrètement, la décision d'exclure des listes de médecins de premier recours les porteurs de deux titres revient à écarter leurs patients des modèles d'assurance fondés sur ces listes. Et si ces patients décident malgré tout d'opter pour un de ces modèles - ce qui, en cette période de l'année, doit être le cas de nombre d'entre eux -, ils sont alors contraints de changer de médecin de famille.

Une situation pour le moins absurde car, en cas de changement de médecin, c'est toute la relation de confiance qui doit être rétablie, sans oublier que le nouveau médecin doit tout d'abord se familiariser avec le dossier médical de son patient au prix de plusieurs consultations. La FMH est alors en droit de demander combien coûtent réellement ces changements forcés.

\section{La perte de savoir induite par le changement de médecin de famille peut conduire à une augmentation des coûts.}

L'attitude des assureurs n'est pas non plus défendable sur le plan politique. N'oublions pas que notre pays subit actuellement une pénurie de médecins de famille! Au lieu de restreindre l'accès à la médecine de famille, les caisses feraient donc mieux de tout mettre en œuvre pour créer des modèles encourageant la médecine de premier recours et la qualité des soins. Grâce à leurs connaissances étendues et à leur expérience pluridisciplinaire, les médecins de famille porteurs d'un deuxième titre sont en mesure d'éviter les consultations supplémentaires et donc de réduire les coûts. En outre, les écarter des modèles de médecin de famille risque de les dissuader de participer au service d'urgence. Pouvons-nous vraiment nous le permettre?

Sur le plan juridique enfin, l'exclusion des porteurs de deux titres pose problème en ce sens qu'elle est arbitraire et contraire au principe de l'égalité de traitement. La FMH s'oppose donc vivement aux modèles fondés sur des listes établies exclusivement par les caisses et recommande des modèles reposant sur le partenariat.

Jusqu'ici, j'ai toujours cru au bien-fondé du savoir et de la formation et à la nécessité de les encourager, notamment parce qu'ils représentent des facteurs d'épanouissement personnel essentiels et qu'à ce titre, ils contribuent durablement au développement d'un pays. Pourtant, force est de constater que pour nous, médecins, il vaut mieux ne pas posséder trop de connaissances si nous ne voulons pas être pénalisés...

Dr Jürg Schlup, président de la FMH 